\title{
The Morphologic-Aware Self Adaptive (MASA) System with Image Analysis Technique for Zoysiagrass Managements
}

\author{
Yong-Ik Kwon $^{1 \dagger}$, Jeong-Beom Park ${ }^{2 \dagger}$, Seung-Woo Nho ${ }^{2}$, Yong-shin Kim², Sang-Won Lee, Jin-Woo Eo*, \\ Hyo-Yeon Lee ${ }^{1 *}$ \\ ${ }^{1}$ Subtropical Horticulture Research Institute, Jeju National University, Jeju 63243, Korea \\ ${ }^{2}$ Department. Intelligent System Development EMETIS Co., Ltd., Tokyo 105-0003, Japan \\ ${ }^{3}$ Department Realistic Media Platform Research Center, Korea Electronics Technology Institute, Seongnam 13509, Korea \\ ${ }^{4}$ Department Electronics and Electrical Engineering Dankook University, Yongin 16891, Korea
}

\begin{abstract}
Zoysiagrass (Zoysia japonica Steud.) is one of the most popularly cultivated grasses for sports and recreational environments, particularly in warm region. Zoysiagrass has a tardiness of vegetative propagation compared to other turfgrass. Thus farmers and green keepers want to quickly recognize the field information for management. We discuss the design of a Morphologic-Aware Self-Adaptive (MASA) system for zoysiagrass agricultural business. MASA system with image analysis technique is able to rapidly detect grass blade color and length variations caused by changing climate and environment stress. In the future, farmers and green keepers will be able to have smart-farming and smart-management with MASA system.
\end{abstract}

Keywords Zoysiagrass, Morphologic-aware self adaptive (MASA) system, Image analysis technique, Wireless Sensor Networks (WSNs)

\section{INTRODUCTION}

The turfgrass industry in the world continues to grow due to demand for residential and commercial property development, and the recreational environmental benefits of turfgrass in the landscape. The turfgrass provides safe playing surfaces on athletic fields, outdoor recreation for golfers on golf courses. The turfgrass is a $\$ 40$ billion, 50 million acre industry in the USA (NTF 2016). The zoysiagrass, called Japanese lawn grass or Korean lawn grass (the major warm-season turfgrass), use in garden, landscape and on golf courses (using in tee and fairways) has primarily occurred in the transition zone of the United States (Emmons 2015). Zoysiagrass breeding and development program is in its 12 th year of funding through the United States Golf Association. Considerable genetic variability does exit among Zoysia spp. and after many years of selections, evaluations, and extensive examinations (Yeam et al. 1984). The zoysiagrasses are perennial species that possess both stolon and rhizome. The grasses turn yellow after the first hard frost and are among the first warm season grasses to green up in the spring. The color and shape of grasses leaf blades are very important quality (Bae et al. 2010). Variation associated with several abiotic, biotic, and a turfgrass performance characteristic is well documented. In laboratory, glasshouse, and field studies utilizing zoysiagrass were conducted sting nematode (Belonolaimus longicaudatus) response, effects

Received October 17, 2016; Revised November 25, 2016; Accepted November 25, 2016; Published November 30 , 2016

*Corresponding author Jin-Woo Eo, jweo@dankook.ac.kr, Tel: +82-31-8005-3632, Fax: +82-31-8021-7211

Hyo-Yeon Lee, hyoyeon@jejunu.ac.kr, Tel: +82-64-754-3985, Fax: +82-64-725-0989

${ }^{\dagger}$ These authors contributed equally to this work. 
of nitrogen fertilization rate and mowing height management, and broad-sense heritability estimates of turfgrass performance and stress related characteristics (Lee et al. 2013). The study of turfgrass is well documented but, nonetheless, farmer and green keeper have difficulties with management. A farmer and green keeper sometimes miss a damaged spots on the extensive turfgrass field. The missing damaged spots eventually present economic loss to turfgrass farmer and the field of users.

Nowadays, the smart-farming system assists farmer with speediness and correctness. The smart-farming system has contributed significantly to the success of farming. A various sensors communicate in the field or greenhouse. The communicated information from sensors has been delivered analyzing system through network line. Finally, farmers receive analyzed data from smart-farming system. This study is to optimize Morphologic-Aware Self Adaptive (MASA) System for zoysiagrass.

This paper is organized as follows. 'Materials and Methods' gives an overview of existing approaches to sensing materials and method using spectral and geometric information and its application to collaborative WSNs. 'Approaches to steps proposed method' describes overall system implementation on our sensing devices including the design of a MASA scheme for zoysiagrass agricultural business, MASA. By regulating the mesh sensor network deployment and formulating a collaborative mechanism based on a regular morphologic strategy. 'Results' describes result and how improved tips into system level performance gains. Finally we conclude and discuss future work.

\section{MATERIALS AND METHODS}

\section{Wireless sensor networks (WSNs)}

Wireless communication system and WSNs particularly, are becoming widely adopted and are spread in many applications. Due to its flexibility we have been using wireless technologies in agricultural environments for a long time. Recently wireless sensors are becoming more and more used in agricultural applications. We intend to use it in agroforestry environments, not only to transport data from sensors (spread in the grass field or carried by planting workers and breeding owners), but also to detect blade color and length for the breeding of zoysiagrass (Hyun et al. 2012).

In the present work an Institute of Electrical and Electronics Engineers (IEEE) 802.15.4 and IEEE 802.11ac based network is used. ZigBee and Wi-Fi, which is one standard with a large adoption by the wireless sensor communication network developers community, operates on top of IEEE 802.15.4 and IEEE 802.11ac (ZigBee Alliance 2008). Both protocols have in fact an increasing importance in cooperative WSN implementation, and ZigBee and Wi-Fi, which adds the upper layers of the protocol stack, is considered the most promising standard for wireless sensors (Wang et al. 2006).

Besides low resource end-devices, where sensors are connected, IEEE 802.15.4 and IEEE 802.11ac, ZigBee, Wi-Fi define the existence of devices with more resources than end-devices, that act as network coordinators (ZigBee and IEEE 802.15.4) or Trans-routers (ZigBee to Wi-Fi). Those devices are the WSN infrastructure, which allows data to flow between source and destination nodes. This network infrastructure can also be used to act as a sensing element, since they have fixed geographical position, they can be used to measure the blade color and length, breeding environment components for zoysiagrass growth between wireless nodes.

\section{The nodes of WSNs}

Our present WSNs enable simultaneous, high-speed sensing and data acquisition from multiple wireless wind gauges, anemometer, and intensity of illumination, temperature, humidity and camera inputs. Our range of wireless sensing systems are ideal for both small scale applications requiring a few sensor nodes and large scale applications requiring hundreds of sensor nodes.

Wireless sensor nodes offer a streamlined solution for remote and embedded monitoring that eliminates the need to install or maintain wires. Our wireless nodes support a wide range of sensor types and packaging options, allowing users to quickly configure their optimal network. Furthermore, low-power designs are coupled with internal batteries, and optional energy harvesters, for long-term 
application.

Illumination intensity sensor use programmable lightto-frequency converters combine a configurable silicon photodiode and a current-to-frequency converter on single monolithic short for complementary metal-oxide semiconductor (CMOS) integrated circuit. The output can be either a pulse train or a square wave with frequency directly proportional to light intensity. Camera sensor use CMOS camera module include the active array size of $640 \times 480$, maximum image transfer rate of $60 \mathrm{fps}$ for VGA Auto focus.

\section{The field of zoysiagrass}

Proposed study was conducted on a test field located in Seogwipo city, Jeju Province, Republic of Korea. The test field consisted of plot with zoysiagrass (Fig. 1). The test field already is grown with over 5-year-old zoysiagrass (Zoysia japonica Steud.).

\section{The planting of zoysiagrass (Zoysia japonica Steud.)}

The zoysiagrass was vegetatively propagated in Jeju National University-approved confined green house as well as in a test field in Jeju National University zoysiagrass field. For propagation analysis, sod is cut into small pieces called plugs $(10 \times 10 \mathrm{~cm})$. The plugs are planting on pots $(30 \times 30 \mathrm{~cm})$. The zoysiagrass cultivated according to modified common methods for morphological measurement (Crippen 1990). Watering is dependent on season, geography, but normal rates of watering are one per week. Mowing intervals are 7 to 10 days during the early stage of growth period (from April to June) for synchronization of growing stage.
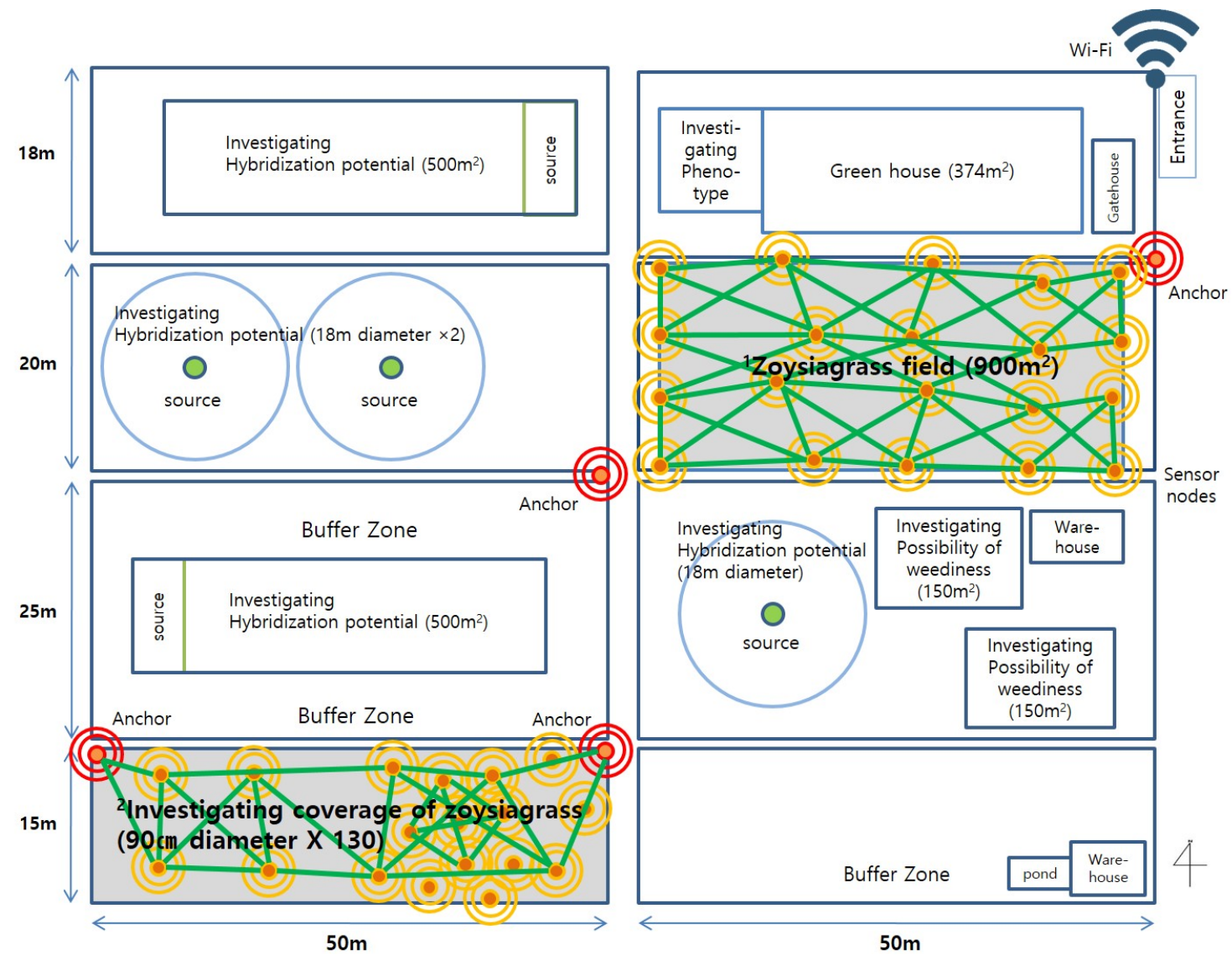

Fig. 1. Map of Jeju National University zoysiagrass field. The gray boxes are test field for this study. ${ }^{1}$ The test field for Morphologic-Aware Self Adaptive. ${ }^{2}$ The area of propagation analysis. Yellow circles are sensor nodes, red circles are anchors. 


\section{Approaches to steps of proposed method}

The MASA method consists of four steps. In the first, proposed study combines two complementary approaches to blade color detection and blade length detection that have long been used in the image processing. Secondly, captured zoysiagrass image is smoothed by median filter to suppress unwanted noise in the image. Thirdly, maximum directional differences of sum of gray values (Red+Green+ Blue, RGB) are calculated for each pixel. In the final step, image is thresholded with a single threshold value and finally the detected edges are thinned to get the proper edge map (Camilli et al. 2007).

\section{Morphologic-aware strategy}

The blade color reveals damage by abiotic and biotic stress. The damaged blade turns to yellow or brown from green. It is considered that damaged plug is detection of over $30 \%$ changed blade color. In a field, even if detects changing blade color only small area, farmer should be control the damage. MASA can detect the changing blade color. Zoysiagrass generally should be mowed to a height of 2 to $3 \mathrm{~cm}$ from the ground. MASA detect the length of blade $(>3 \mathrm{~cm})$ and alert the farmer or green keeper to mow. Mowing frequency varies based on the use and location. For example, a golf course typically mows its golf greens on a daily basis, while gardener mows a roadside several times a year. MASA also is able to customize the alert to use of grass.

\section{Directional color difference calculation}

Captured color image is analyzed from RGB color space point of view. Each pixel in the image consists of three color channels known as RGB components. The range of values of each of this components lies within 0 to 255 . Edges exist in a color image where abrupt changes of RGB values occur. Therefore, to detect proper edges, first the abrupt color differences in an image must be pointed out. To reduce the computational overhead we have calculated a transformed value for each pixel which converts three component valued pixels into a single valued attribute (Naik and Murthy 2006). Using the weighted sum technique, we can easily overcome this type of problem and can calculate exact color differences using four masks of $3 \times 3$.

\section{Threshold technique}

Threshold technique is very important task in color edge detection schemes. The accuracy of a scheme is dependent on the choice of threshold parameters. One of the foremost criteria of thresholding is that the program should be efficient enough to automatically compute the optimum threshold parameter. The criteria of selection of a parameter for a given image are that the resultant edge map should satisfy the following: 1) It should contain most of the prominent edges; 2) It should not contain too much spurious edges; 3) It should be meaningful and visibly pleasing (Dony and Wesolkowski 1999).

\section{Thinning scheme}

Edge map produced in this way contains thick edges. Thus, a thinning technique is applied to create more thin edges which will be more accurate and visibly soothing. Using two $3 \times 3$ masks, simple edge thinning scheme is used to extract accurate edge.

\section{RESULTS}

As stated in the previous section, to validate the proposed blade color and length detection based on MASA method, several measurements were made in a real zoysiagrass field scenario. Those in-field measurements included information measurements of the breeding and propagation for zoysiagrass with and without camera sensor and growth environment measuring sensors, with blade color and length detection and transmitting to application the data after calculating ideal mowing point from leaf's morphology, simulating zoysiagrass planting environments, to evaluate the relation between blade color and length detection of zoysiagrass and the sensor placement.

The first set of measurements was taken with the CMOS camera sensor at the zoysiagrass field and proliferating plugs. It included the detection measurement of blade color in the two specified areas types. Results from this set of measurements are shown in Fig. 2A-C, correspond to 

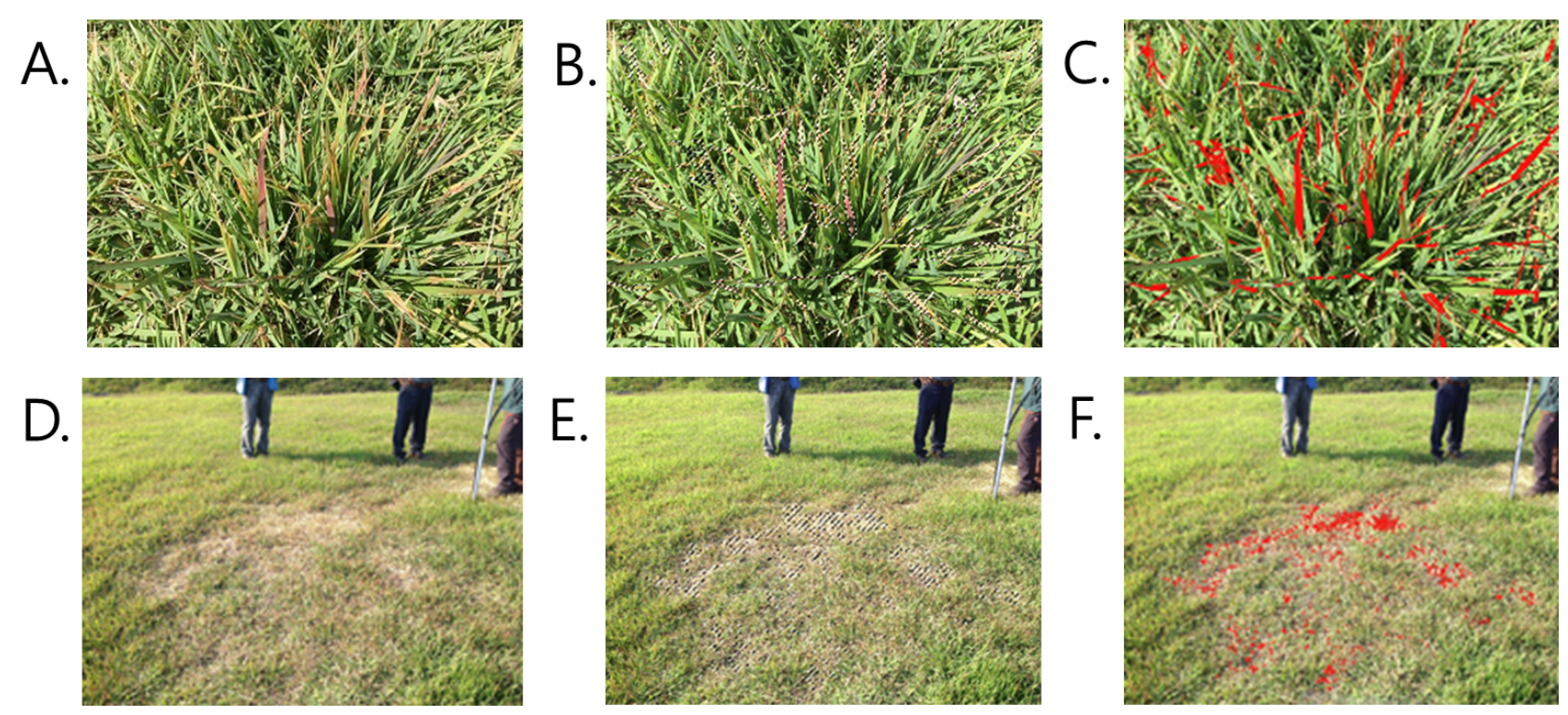

Fig. 2. Detection of changed blade color on the proliferating plug (A-C) and in the field (D-F). (A, D) Still pictures, (B, E) recognition of blade edge by scaling and filtering, (C, F) detection of changed blade color. The blade color reveals damage by abiotic and biotic stress. The damaged blade turns to yellow or brown from green. It is considered that damaged plug is detection of over $30 \%$ changed blade color. In a field, even if detects changing blade color only small area, farmer should be control the damage. MASA can detect the changing blade color.
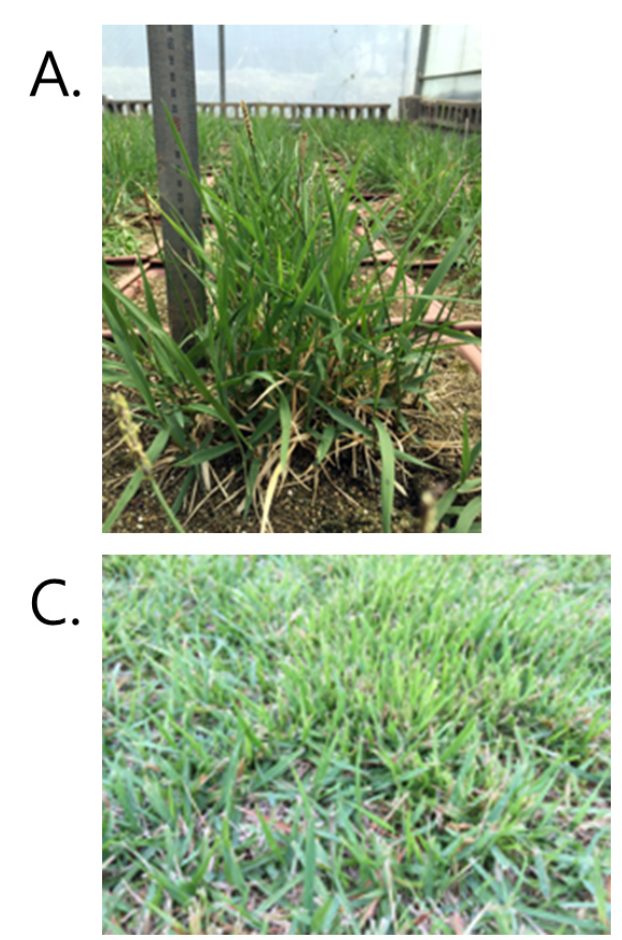
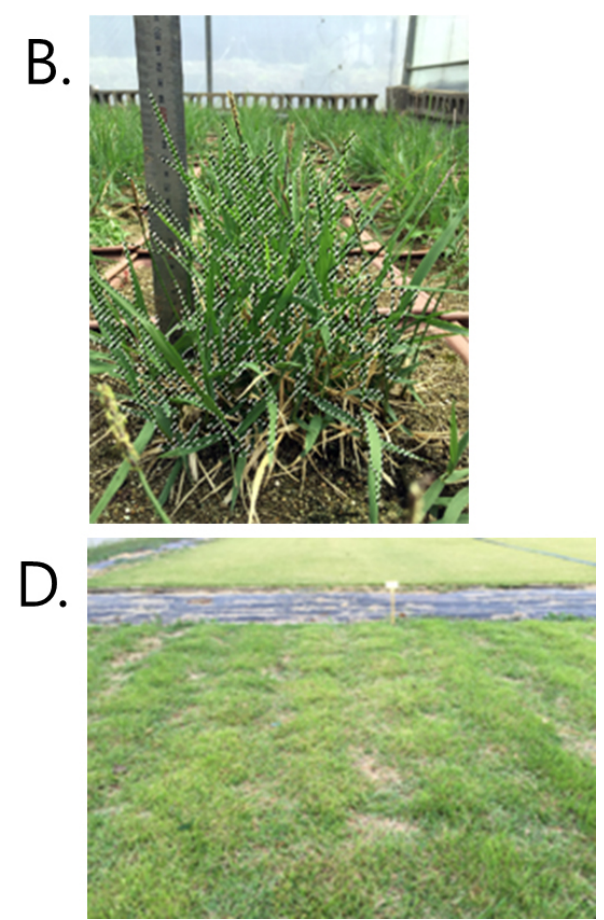

Fig. 3. Detection of blade length on the plug (A, B) and mowed zoysiagrass field (C, D). Morphologic-Aware Self Adaptive (MASA) method detected blade length on the plugs (B). The leaf blade tips of mowed zoysiagrass were blunt end (C). Zoysiagrass generally should be mowed to a height of $2 \mathrm{~cm}$ to $3 \mathrm{~cm}$ from the ground. MASA detect the length of blade $(>3 \mathrm{~cm})$ and alert the farmer or green keeper to mow. MASA also is able to customize the alert to use of grass. 
measuring blade color detection on the cultivation stage, where as expected the lower detection values were found. The Fig. 2D-F correspond to the blade color detection in a high-density zoysiagrass field, where the higher attenuation was detected.

Results from the second set of measurements are presented in Fig. 3. The blade length measurement, were taken in the low-density propagation and cultivated plugs area, at different distances from the zoysiagrass field. The Fig. 3A corresponds to the analog measurement in plugs, at investigating coverage of zoysiagrass. The Fig. 3B corresponds to measuring blade length detection in plugs. The Fig. 3C, D. correspond to the after ideal mowing work and at zoysiagrass field. Zoysiagrass grows a maximum over $20 \mathrm{~cm}$ in natural environment. A healthy zoysiagrass should be mowed to a height of $2 \mathrm{~cm}$ to $3 \mathrm{~cm}$ from the ground in a field. However, the height of turfgrass depends on the purpose and turfgrass species.

\section{DISCUSSION}

The valuable information is supporting easily and quickly to a farmer, since information \& communication technology (ICT) fuses with biotechnology to begin the smart-farming. Recently, the smart-farming system is able to detection of borer insects in tomatoes (Rupanagudi et al. 2015). In Korea, smart-farming technology almost was applied to greenhouse. The smart greenhouse system reduces management time and resources, and increases products, though the some parts of system are overseas product which is costing too much to install and maintain (Kim and Huh 2015). The smart-farming technology is a beginning stage in Korea. Thus, this study will improve a smart-farming technology and not introduce in greenhouse but in field. Because, smart-farming focuses almost greenhouse, whereas ICT not yet endeavors zoysiagrass field.

The proposed study represents a prototype application of MASA method based on image analysis and WSNs system for turfgrass. The aim of this analysis is to reduce labor of turfgrass cultivation and management by proposed agricultural MASA method. The results reported here indicate that MASA method could detect changed blade color on the plugs and in the field. Also, it detected blade length on the plugs. In this time, the MASA system get perceive changed condition of zoysiagrass for report to farmer, though our sensor nodes will have provided the surface mounted temperature and humidity, intensity of brightness, visual environmental information. Finally, the MASA application provides management information for farmers and green keeper from both the environmental information and the morphological character analysis.

In the near future, we will improve novel MASA method to detect all turfgrass of morphological character in the field. The proposed method may be handy for any computer vision task where extraction of edge maps is required for a large set of images for feature extraction or for any other work. Our next venture will be comparing those algorithms with the proposed one and analyze the performance on the basis of parameters like computing time, execution complexity and accuracy of the system output in presence of noise.

\section{ACKNOWLEDGEMENTS}

The Authors would like to thank the EMETIS Co., Ltd. for their support of this study through image processing scheme and the WSNs technology. This work was carried out with the support of Cooperative Research Program for Agriculture Science \& Technology Development (Project No. PJ011280), Rural Development Administration, Republic of Korea. This research was supported by Basic Science Research Program through the National Research Foundation of Korea (NRF) funded by the Ministry of Education (2016R1A6A1A03012862).

\section{REFERENCES}

Bae EJ, Park NC, Lee KS, Lee SM, Choi JS, Yang GM. 2010. Distribution and morphology characteristics of native zoysiagrasses (Zoysia spp.) grown in South Korea. Asian J. Turfgrass Sci. 24: 97-105.

Camilli A, Cugnasca CE, Saraiva AM, Hirakawa AR, Corrêa 
PLP. 2007. From wireless sensors to field mapping: anatomy of an application for precision agriculture. Comput. Elect. Agric. 58: 25-36.

Crippen RE. 1990. Calculating the vegetation index faster. Remote Sens. Environ. 34: 71-73.

Dony RD, Wesolkowski S. 1999. Edge detection on color images using RGB vector angles. vol. 2. Proc. of the: Electrical and Computer Engineering, 1999 IEEE Canadian Conference on. IEEE Hoboken, NJ. pp.687-692.

Emmons RD. 2015. Turfgrass science and management. 5th ed. Cengage Learning, Stamford, CT.

Hyun YH, Choi BJ, Kim YJ, Joo YK. 2012. Analysis of research trend on zoysiagrass (Zoysia spp.). Asian J. Turfgrass Sci. 26: 89-95.

Kim GJ, Huh JD. 2015. Trends and prospects of smart farm technology. Elect. Telecommun. Trends 30: 1-10.

Lee S, Yu HC, Yoon BS, Yang GM, Kim JY, Kim Y, et al. 2013. Soil and morphological characteristics of native zoysiagrasses by the habitats. Weed Turf. Sci. 2: 55-61.

Naik SK, Murthy CA. 2006. Standardization of edge magnitude in color images. IEEE Trans. Image Process. 15: 2588-2595.

NTF. 2016. National Turfgrass Federation [Internet]. USDA, Washington, DC. [cited 2016 May 27]. Available from: http://www.turfresearch.org/pdf/Industry\%20Turf\%20I nitiative.pdf.

Rupanagudi SR, Ranjani BS, Nagaraj P, Bhat VG, Thippeswamy G. 2015. A novel cloud computing based smart farming system for early detection of borer insects in tomatoes. Proc. of the: Communication, Information \& Computing Technology (ICCICT), 2015 International Conference on, IEEE Hoboken, NJ. pp.1-6.

Wang N, Zhang N, Wang M. 2006. Wireless sensors in agriculture and food industry-Recent development and future perspective. Comput. Elect. Agric. 50: 1-14.

Yeam DY, Hong KH, Han IS. 1984. The relation between leaf-node stage and flower initiation in zoysia species. J. Korean Soc. Hort. Sci. 25: 182-185.

ZigBee Alliance. 2008. ZigBee-2007 Specification, Document 053474r17. ZigBee Alliance, Davis, CA. 